\title{
Betriebliche Weiterbildung in Klein- und Mittelbetrieben (KMU). Forschungsstand, Problemlagen und Handlungserfordernisse. Eine Bilanz
}

Kleine und mittlere Unternehmen (KMU) bilden das beschäftigungspolitische Rückgrat der deutschen Wirtschaft. Sie sind nicht nur technologischer Innovationstreiber, sondern auch das Herzstück beruflicher Ausbildung. In der beruflichen Weiterbildung sind sie jedoch deutlich zurückhaltend. Der Beitrag liefert eine Bestandsaufnahme auf Basis von Forschungsergebnissen und fragt, welche spezifischen Umsetzungsprobleme bei der Entwicklung von beruflicher Weiterbildung entstehen und inwieweit die Intensivierung einer auf KMU zugeschnittenen Qualifizierungsberatung Lösungsansätze für ein höheres betriebliches Weiterbildungsengagement liefern kann.

ROLF DOBISCHAT, KARL DÜSSELDORFF

\section{Problemstellung}

Die Relevanz der betrieblichen Weiterbildung ist in ihren unterschiedlichen Funktionen und Aufgaben weitgehend unumstritten. Bei den Begründungen wird auf demografische, arbeitsmarktpolitische wie auch berufsstrukturelle Veränderungsprozesse im Kontext des prognostizierten Fachkräftebedarfs verwiesen. Betrachtet man die Daten zur Weiterbildungsteilnahme der Bevölkerung im Zeitverlauf, so ist nach einer Stagnationsphase ein deutliches Ansteigen zu diagnostizieren, ${ }^{1}$ wobei die sozialen Disparitäten in der Teilnehmerstruktur anhand der bekannten bildungssoziologischen Koordinaten (Status, Einkommen, formales Bildungsniveau u. a.) weiterhin stabil sind (Autorengruppe
Bildungsberichterstattung 2012, S. 155). Nach „Segmenten differenziert" ist im allgemeinen Aufwärtstrend auch die Partizipation an der betrieblichen Weiterbildung gestiegen (BMBF 2013, S. 21).

Im Folgenden soll eine Bilanzierung vorgenommen und thematisiert werden, wie sich die betriebliche Weiterbildung in Klein- und Mittelbetrieben (KMU) ${ }^{2}$ in der Vergangenheit entwickelt hat, mit welchen Problemen KMU bei der Initiierung von Weiterbildung konfrontiert sind und wo ein möglicher Handlungs- und Gestaltungsbedarf identifiziert werden kann. Der Fokus auf den Bereich der KMU liegt deshalb nahe, weil sie quasi das Rückgrat der Betriebe in Deutschland sind und in diesem Sektor der überwiegende Teil der sozialversicherungspflichtigen Personen tätig ist. ${ }^{3}$

\footnotetext{
1 Nach den Ergebnissen des Adult Education Survey stagniert die Teilnahmequote der 19- bis 64-jährigen Bevölkerung auf einem Niveau von ca. 42 \% (2010). Der Rückgang in derTeilnahmequote in den Jahren 2007 bis 2010 ist auf das rückläufige Volumen der betrieblichen Weiterbildung zurückzuführen (Autorengruppe Bildungsberichterstattung 2012, S. 142). Nach den jüngsten Ergebnissen des AES 2012 (BMBF 2013, S. 21) ist die Teilnahmequote an der beruflichen Weiterbildung im Vergleich der Jahre 2010 und 2012 von 26 auf $35 \%$ gestiegen

Sichtweise zählen Betriebe mit einer Zahl bis zu neun Beschäftigten zu den kleinen und Betriebe von zehn bis zu 499 Beschäftigten zu den mittleren Unternehmen. Differenzierter hat die EU (2005) die Betriebsgröße klassifiziert, in dem zwischen Mikro (ein-neun Beschäftigte), Klein (zehn-49 Beschäftigte), Mittel (50-249 Beschäftigte) und Groß (250 und mehr) unterschieden wird. Ein weiteres Kriterium bei der Abgrenzung der Betriebsgröße bildet der jährlich erzielte Umsatz. Neben quantitativen MerkmaIen lassen sich auch qualitative Merkmale für eine Unterscheidung heranziehen. So z. B. der stark personengepräg te Charakter der Betriebsführung wie auch die Eigentumsverhältnisse. 


\section{Weiterbildungsaktivitäten und -hemmnisse bei KMU}

\subsection{Hemmfaktoren}

Repräsentative Strukturdaten zur betrieblichen Weiterbildung werden seit Jahren in bestimmten Zeitabständen durch empirische Untersuchungen erhoben. ${ }^{4} \mathrm{Nach}$ den aktuellen AES-Ergebnissen (BMBF 2013, S. 20) liegt die Weiterbildungsteilnahme der Bevölkerung im Jahr 2012 bei $49 \%$. Die Teilnahmequote an betrieblicher Weiterbildung bewegt sich auf einem Niveau von $35 \%$. Wie durch Studien immer wieder belegt, besteht eine enge Korrelation zwischen der betrieblichen Weiterbildungsaktivität und der Zahl der Beschäftigten. Der Effekt der Betriebsgröße ${ }^{5}$ auf das betriebliche Weiterbildungsverhalten spiegelt sich auch erneut in den jüngsten AES-Daten wider. Mit ansteigender Betriebsgröße geht ein kontinuierliches Ansteigen der Weiterbildungsbeteiligung unter den Beschäftigten einher. Während für Großbetriebe mit über 1.000 Beschäftigten eine Beteiligungsquote von $63 \%$ registriert wird, liegt die Quote bei Kleinbetrieben mit bis zu zehn Beschäftigten mit $36 \%$ deutlich darunter.

Während festgestellt werden kann, dass mit der Größe des Beschäftigungsbetriebs durchaus unterschiedliche Chancenzugänge zur betrieblichen Weiterbildung verbunden sind, bleiben andere Faktoren, die auf die Zugangschancen ebenfalls einwirken, davon relativ wenig beeinflusst. So haben die bekannten selektiven Partizipationswirkungen, die in Abhängigkeit bestimmter intervenierender Faktoren (Status, Qualifikationsniveau, Alter, Einkommen etc.) stehen und die markanten und stabilen Beteiligungsmuster bzw. soziale Disparitäten hervorbringen, nach wie vor weit- gehend Bestand. (BIBB 2012, S. 295; BMBF 2013; Dobischat et al. 2011).

Worin liegen also die Gründe, warum KMU weniger in Weiterbildung involviert sind? Für Staudt (1993, S. 137) sind die Ursachen darin zu sehen, dass in vielen Betrieben Weiterbildung eine weitgehend isoliert vom normalen Betriebsablauf stehende Aktivität ist. Weiterbildung gilt als ein „kostenaufwendiges“, den „,normalen Produktions- und Dienstleistungsprozess störendes“, „zusätzlichen Abstimmungsprozessen unterworfenes" und „besonderen Rechtfertigungen unterlegenes“ Unterfangen (Staudt 1993, S. 137). Diese skeptische Einschätzung stellt neben den organisatorischen Problemen vorrangig auf die Kostenseite von Qualifizierung ab, die ja eine betriebliche Investition in die „Humankapitalausstattung“ darstellt. ${ }^{6}$ Da sich die Investitionen infolge unzureichender Verfahren einer Erfolgsmessung durch ein kurzfristig Auskunft gebendes Bildungscontrolling nicht darstellen, sondern sich erst in längerfristigen Zeithorizonten hinsichtlich der Effizienz und der Erträge bewerten lassen, tragen Investitionen in die Weiterbildung der Beschäftigten eine hohe risikoinduzierte Belastung (Neubäumer et al. 2006), was zu Unsicherheit führt, ob sich die Investitionen in einem überschaubaren Zeitraum amortisieren oder ob die Erträge im Worst Case sogar durch Abwanderung von qualifiziertem Personal externalisiert werden. Eine derartige Zurückhaltung gegenüber betrieblichen Bildungsinvestitionen verstärkt sich dann, wenn ein harter Wettbewerb mit Konkurrenten gegeben und die Ertragslage nicht ausreichend ist. Dass die Ertragslage wie auch die Innovationsaktivität ${ }^{7}$ wichtige Einflussfaktoren beim Engagement in der Weiterbildung darstellen, wird durch Daten des IAB-Panels belegt. Demnach veranlasst eine gute Ertragslage eine höhere Weiterbildungsaktivität, was insbesondere bei kleinen Betrieben sichtbar wird (Autorengruppe Bildungsberichterstattung 2012, S. 145). ${ }^{8}$
3 Nach Berechnungen des Instituts für Mittelstandsforschung (IfM 2011) gibt es ca. 3,6 Mio. KMU in Deutschland, was bedeutet, dass 99,7\% aller steuerpflichtigen Unternehmen KMU sind, die ca. 20 Mio. sozialversicherungspflichtige Personen beschäftigen, was einem Anteil an der Gesamtbeschäftigung von 79,5 \% entspricht. Nahezu die Hälfte aller KMU-Beschäftigten arbeitet in Betrieben mit weniger als 50 Personen (Möller/Walwei 2009, S. 366).

4 Dabei handelt es sich um folgende Studien: die europäisch vergleichende CVTS (Continuing Vocational Training Survey), das Betriebspanel des IAB (Institut für Arbeitsmarkt- und Berufsforschung), die Weiterbildungserhebung des IW (Institut der deutschen Wirtschaft) und die ebenfalls europäisch vergleichende Erhebung Adult Education Survey (AES), die im Auftrag des BMBF durchgeführt wird und das Berichtssystem Weiterbildung (BSW) abgelöst hat. Zu berücksichtigen ist dabei, dass eine Vergleichbarkeit zwischen den erhobenen Daten aus methodischen Gründen nur bedingt möglich ist (Käpplinger 2007, S. 383).

5 Bei der Kategorie Betriebsgröße ist auf ein methodisches Problem hinzuweisen. In statistischen Erhebungen wird die Weiterbildungsteilnahme in Abhängigkeit von der Be- triebsgröße ausgewiesen, wobei die Quote in einzelnen Betriebsstätten erhoben wird. Eine Erfassung der Quoten auf Unternehmensebene mit der Subexistenz vieler Betriebsstätten kann zu statistischen Verzerrungen in der Quote in Abhängigkeit vom Zugriff führen.

6 In der Humankapitaltheorie wird das ökonomische Rationalitätsprinzip auf das Bildungsverhalten übertragen. Eine Bildungsinvestition gilt dann als ökonomisch rational, wenn durch Bildung und Qualifikation Erträge erwirtschaftet werden, die die Investitionen übersteigen und zu einer messbaren Rendite führen.

7 Unter Innovationsaktivität wird die Produktverbesserung, die Produkterweiterung sowie neue Verfahren zur Verbesserung des Produktions- oder Dienstleistungsprozesses verstanden (IAB, Betriebspanel, Tab G2-7web im Anhang zum Bildungsbericht, Autorengruppe Bildungsberichterstattung 2012).

8 Die detaillierten Daten im Zeitverlauf von 2003 bis 2010 können derTab. G2-6web im Anhang zum Bildungsbericht 2012 (Autorengruppe Bildungsberichterstattung 2012) entnommen werden. 
Die Innovationsaktivität als ein weiteres auslösendes Moment für Weiterbildung und Qualifizierung schlägt sich vornehmlich in mittleren und größeren Betrieben nieder, Kleinbetriebe hingegen bewegen sich in ihrer Aktivität unterhalb des prozentualen Durchschnitts aller Betriebsgrößenklassen. ${ }^{9}$ Ein deutlicher Zusammenhang zwischen Betriebsgröße und Weiterbildungsaktivität existiert beim Merkmal „Ausbildungsbetrieb“. Kleinbetriebe (ein bis 19 Beschäftigte), die eine berufliche Ausbildung anbieten, verzeichnen gegenüber Betrieben, die nicht in der beruflichen Ausbildung engagiert sind, eine bemerkenswert höhere Weiterbildungsbeteiligung (71,2 zu 49,4 \%). Dieser positive Effekt, der durch das Merkmal „Ausbildungsbetrieb“ verursacht ist, schwächt sich jedoch mit wachsender Betriebsgröße deutlich ab. Bei Großbetrieben mit über 200 Beschäftigten ist deren hohes Weiterbildungsengagement unabhängig von der Tatsache, ob sie berufliche Ausbildung betreiben oder nicht (BIBB 2012, S. 302). Als weiteres beeinflussendes Merkmal auf die Weiterbildungsaktivität ist anzusehen, dass das Aktivitätsniveau bei Betrieben mit Personalbedarf erheblich höher liegt als bei Betrieben, die keine Rekrutierungsprobleme aufweisen. Kleinbetriebe mit bis zu 19 Beschäftigten, die Personal durch Stellenausschreibungen suchen, sind in 62,5 \% der Fälle weiterbildungsaktiv, während Betriebe, die keine Personalprobleme artikulieren, nur zu $46 \%$ Weiterbildung aktiv betreiben (BIBB 2012, S. 302ff.).

Aus diesen Ergebnissen wird einerseits deutlich, dass kleinere Betriebe dann höhere Weiterbildungsaktivitäten entwickeln, wenn sie auch in der beruflichen Erstausbildung engagiert sind. Andererseits bietet die vergebliche Suche nach Fachkräften eine hinreichende Stimulanz, die Rekrutierungskosten zu vermeiden und in die eigene Weiterbildung und Qualifizierung zu investieren. Die genannten Einflussfaktoren können somit ein höheres Weiterbildungsengagement von KMU erklären, sodass der vielfach geäußerte pauschale Befund, KMU seien per se weiterbildungsinaktiv, ja sogar gegenüber Qualifizierung und Personalentwicklung defensiv und resistent, zu relativieren ist (Düsseldorff 2006, S. 182). Dennoch, eine wichtige Ursache für die geringere Weiterbildungsbeteiligung von KMU wird in der mangelnden infrastrukturellen Ausstattung und in fehlenden Kompetenzen der mit Weiterbildung beauftragten Mitarbeiter sowie dem geringen Institutionalisierungsgrad von Weiterbildungsplanung und Analysen zum Bildungsbedarf gesehen (Leber 2009; Haak 2003).

Aber selbst wenn Unternehmensverantwortliche den Wert der betrieblichen Weiterbildung erkennen, sind entscheidende Voraussetzungen für eine systematische betrieblich berufliche Weiterbildung in KMU oft nicht gegeben oder nur gering ausgeprägt. So existiert das Problem eines betriebsgrößenabhängigen Institutionalisierungsgrades des Personalwesens und eine damit korrespondierende, nur gering formalisierte Form der Personalplanung und -entwicklung (Kay et al. 2008; Richter 2009) wie auch des fehlenden „Know How“ einer strategisch angelegten Investitionsplanung (Düsseldorff 2006) im Sinne einer Verbindung zwischen Unternehmens- und Personalentwicklung (Kay et al. 2008, S. 133ff.). Insofern ist den KMU zu attestieren, dass sie mehrheitlich nicht über die Ressourcen für notwendige infrastrukturelle, personelle und organisatorische Voraussetzungen verfügen, die erst eine gezielte betriebliche Weiterbildung als Teil einer Personal- und Organisationsentwicklung möglich machen.

Trotz der erheblichen Differenzen beim Institutionalisierungs- und damit Professionalisierungsgrad zwischen größeren und kleineren Betrieben gibt es beim Angebotsspektrum wie auch bei den Lernformen betrieblicher Weiterbildung nur unwesentliche Abweichungen. Sucht man nach Lernarrangements, die von Klein- und Mittelbetrieben besonders häufig eingesetzt werden, so ist festzustellen, dass alle relevanten Lern- und Organisationsformen wie bei größeren Betrieben zur Anwendung kommen, wenngleich die größeren und vor allem die Großbetriebe die Breite des Spektrums an Lernformen intensiver ausschöpfen als kleinere, weiterbildungsaktive Unternehmen. ${ }^{10}$

Auch beim Ranking der Weiterbildungsziele besteht in Abhängigkeit von der Betriebsgröße keine auffällige Abweichung bei der Priorisierung der Ziele. An erster Stelle steht die fachliche Anpassung an veränderte Arbeitsabläufe (83\%), gefolgt von der Förderung des selbstständigen Arbeitens ( $73 \%$ ) und der Verbesserung der kommunikativen Kompetenz (66 \%) (Autorengruppe Bildungsberichterstattung 2012, S. 147, Abb. G2 - 2). Diese Befunde überraschen, denn man kann die Struktur und Organisation betrieblichen Lernens in Großbetrieben nicht als Referenzmodell für die betriebliche Bildungsarbeit in KMU ansehen, da oftmals andere Bildungsstrategien angewendet werden, die eher einem weiten Begriff von Weiterbildung gerecht werden (Gonon et al. 2004; Gonon/Stolz 2004; Elsholz 2010, S. 6). In diesem Zusammenhang ist problematisch, dass KMU nur allzu häufig infolge fehlender Beratung versuchen, die Strategien und Konzepte der großen Betriebe zu kopieren. Sie übernehmen das, was in Fachmagazinen oder auf Online-Portalen als Mainstream steht und hinterfragen nicht, welche betriebsspezifischen Kompetenzen sie als KMU benötigen (Vages 2012).

\subsection{Förderaktivitäten}

Die geschilderten Facetten des Problems, KMU durch mehr selbst initiierte Weiterbildung für mehr Wettbewerbs-

9 Die Daten im Zeitverlauf 2003 bis 2010 können derTab.
G2-7web im Anhang zum Bildungsbericht 2012 (Autoren-
gruppe Bildungsberichterstattung 2012) entnommen werden.

10 Den empirischen Daten der CVTS-Erhebung zufolge zeigen arbeitsplatznahe Lernformen gegenüber formalisierten Kursen für Deutschland im Vergleich zu anderen europäischen Ländern generell eine besondere Relevanz und Aufmerksamkeit (Behringer/Käpplinger 2011, S. 17; Moraal/Schönfeld 2012). 
fähigkeit auszustatten, haben dazu geführt, spezifische politische Förderprogramme aufzulegen, in deren Zielfokus die besondere (institutionelle, organisatorische, finanzielle) Unterstützung und Beratung von KMU bei der Weiterbildung steht (BMBF 2011). Aus der Vielzahl der konzeptionellen Ansätze ist beispielsweise auf die Initiative „Bildungsscheck" des Landes NRW hinzuweisen (Koscheck 2010). Ziel der Initiative ist es, die Investitionsbereitschaft von KMU und ihren Beschäftigten in Qualifikationsmaßnahmen durch finanzielle Anreize zu mobilisieren sowie eine bedarfsgerechte Bildungsberatung und die Kooperation zwischen Betrieben und externen Bildungsdienstleistern zu fördern (Jablonka 2009, S. 45). Die Ergebnisse zeigen, dass durchaus Kleinbetriebe (ein bis neun Beschäftigte) mit dem Programm erreicht wurden, größere Betriebe mit über 100 Beschäftigten aber deutliche Nutznießer der Initiative sind (ebenda, S. 48). Andere staatliche Initiativen wie das über den Europäischen Sozialfonds kofinanzierte Programm mit dem Titel „Lernende Regionen“, das u. a. einen Themenschwerpunkt in der verstärkten Animation von KMU zur Weiterbildung über Netzwerkstrukturen setzte, haben nicht zu den gewünschten nachhaltigen Effekten geführt bzw. den Nachweis einer verbreiteten Effektivität nicht erbringen können.

Die Suche nach überzeugenden Lösungen, wie mittelständische Unternehmen gezielt für die Intensivierung der Weiterbildung unterstützt werden können, hält also an. Neben der Schaffung staatlicher Anreizsysteme können weitere Strategien zur Intensivierung der KMU-Weiterbildung identifiziert werden. Beispiele hierfür sind tarifvertragliche Regelungen der Sozialpartner, die in spezifischen Qualifizierungstarifverträgen ihren Niederschlag gefunden haben (Bahnmüller et al. 2006). Auch dieser Ansatz hat keine nachhaltigen und quantitativ bedeutsamen Markierungspunkte vor allem bei der Schaffung institutionalisierter Beratungsstrukturen setzen können. Zwar hat die Existenz von Qualifizierungsverträgen das Bewusstsein über die Relevanz von Weiterbildung bei den beteiligten Akteuren geschärft und diesbezügliche Aktivitäten ausgelöst, jedoch sind die bisherigen Erfahrungen eher als bescheiden einzustufen. So konnte im IAB-Betriebspanel auf Basis des Indikators „Beteiligungsstruktur“ kein positiver Effekt durch Qualifizierungstarifverträge gemessen werden und auch die soziale Selektivität bei der Teilnehmerrekrutierung blieb weiterhin stabil (Bahnmüller 2012).

Aber auch Förderschwerpunkte wie z. B. das Programm WeGebAU (Weiterbildung Geringqualifizierter und -beschäftigter älterer Arbeitnehmer in Unternehmen), das sich an Betriebe mit einer Belegschaftsgröße unter 250 richtet, hat infolge struktureller Fehlstellungen „den Löwenanteil der für WeGebAU bereitgestellten Mittel für die Förderung von Arbeitslosen verwendet“" (Lott/Spitznagel 2007, S. 3). In der zweiten Phase wurde bilanziert, dass vor allem Kleinstbetriebe und Betriebe mit überwiegend gering qualifiziertem Personal die Angebote nicht oder nur kaum nutzen (Lott/Spitznagel 2010, S. 7). Aus den Ergebnissen kann man ableiten, dass, solange weder KMU durch eine strukturierte Beratung noch die ausgegrenzten Risikogruppen durch eine grundsätzliche, variable und betrieblich wie individuell flexible Anspruchsgrundlage Unterstützung für die Realisierung der beruflich-betrieblichen Weiterbildung erhalten, KMU offensichtlich nicht die betriebsgrößenabhängigen und statusbedingten Beteiligungsungleichheiten in der betrieblichen Weiterbildung nennenswert werden abbauen können.

\section{Qualifizierungsberatung für KMU - ein Königsweg?}

\subsection{Strategische Bedeutung}

In der Debatte über Möglichkeiten, die Weiterbildungsdis$\operatorname{tanz}$ der KMU zu reduzieren, gilt die Qualifizierungsberatung als eine relativ neue richtungsweisende Strategiekomponente. Mit ihr soll das Tableau bekannter Interventionsformen, wie expansivere finanzielle Förderung, Sensibilisierung der KMU gegenüber Weiterbildung als Wettbewerbsfaktor oder Stärkung der öffentlichen Verantwortung durch Regulierung und Infrastrukturentwicklung erweitert werden. Generell ist KMU-Qualifizierungsberatung inzwischen als so genannter kritischer Erfolgsfaktor für die Intensivierung der betrieblichen Weiterbildung in KMU identifiziert und anerkannt (Düsseldorff/Wohlfart 2012, S. 127f.; Loebe/Servering 2012a; Schiersmann 2011, S. 751).

Übereinstimmend wird dabei argumentiert, dass infolge fehlender „Strukturen und Kapazitäten für die systematische Personalentwicklung“ die „Etablierung von Qualifizierungsberatung als Instrument einer zukunftssichernden Organisationsentwicklung“ (Munk 2012, S. 5) als geeignetes und zwingend notwendiges Instrument erforderlich ist. Der Nutzen einer externen Beratung, so wird unterstellt, diene den Betrieben, ihre qualifikatorischen Bedarfe angesichts verschärfter und globalisierter Wettbewerbsbedingungen, demografischer Risiken und technisch bzw. technologisch oder organisational induzierter Anpassungszwänge systematisch zu klären. Erst auf dieser Informationsbasis können betriebliche Bedarfe strategisch und operativ angemessen definiert werden, um dann mit einem angepassten Weiterbildungsverhalten im Sinne von Ausdifferenzierung und Intensivierung reagieren zu können. Mit diesen Prozessschritten ist die Herstellung eines Zusammenhangs zwischen Unternehmensentwicklung, Humankapitalentwicklung und betrieblicher Weiterbildung diagnostisch zu betrachten, um hieraus systematische Qualifizierungsstrategien für unterschiedliche Belegschaftsgruppen als wettbewerbssichernde Interventions- und Investitionsstrategien abzuleiten.

Betrachtet man allerdings den Realisierungsverlauf der oben genannten strategischen Option, eine Intensivierung und qualitative Entwicklung der betrieblichen Weiterbildung 
durch Beratung zu unterstützen, bleibt der Verdacht, beim oben genannten erzielten Konsens handelt es sich um eine vielfältig wiederholte Akklamation, nicht aber um eine Positionsbestimmung, mit der gleichzeitig auch eine nachhaltige Praxis korrespondiert. Dabei gäbe es, wie die idealtypisch aufgelisteten Leistungsmerkmale und Handlungsfelder der KMU Qualifizierungsberatung zeigen, gute Gründe, sie als öffentlich verantwortetes Beratungs- und Unterstützungsangebot für mittelständische Unternehmen zu etablieren, zu finanzieren und qualitativ abzusichern.

\subsection{Forschungsstand}

Ein Blick auf die Forschungslandschaft zeigt, dass Bildungsund Qualifizierungsberatung für KMU „als Forschungsthema [...] in Deutschland nicht auf eine etablierte Forschungstradition zurückgreifen kann“" (Dobischat et al. 2008b, S. 3). Weder fundierte Theoriebildungsprozesse in einem ungeklärten bzw. weitgehend offenen Gegenstandsfeld noch pragmatische, praxisbezogene Forschungsansätze und -erträge sind vorfindbar. Zwar lässt sich zunehmend eine verhaltene Forschungsintensivierung feststellen (Döring/Rätzel 2007a und 2007b; Loebe/Severing 2008; Arnold/Mai 2009; Döring/Hoffmann 2012), aber von einer nachhaltigen Forschungskonsolidierung kann nicht gesprochen werden. Fasst man im Forschungskontext kursorisch den aktuell vorliegenden Status zusammen, kann zwischenbilanziert werden:

- Eine Bildungsberatungsinfrastruktur mit einem eindeutigen Profil in Gestalt einer markierbaren KMU-Qualifizierungsberatung existiert in Deutschland im Vergleich zu anderen OECD-Staaten nicht (Rambóll Management 2007). - Die Identifikation eines Anbieter- oder Nachfragemarktes sowie eine damit verbundene Leistungsdefinition, eine Profilbildung oder eine Typisierung von Nachfragern oder von Anbietern ist momentan nur eingeschränkt möglich (Loebe/ Severing 2011; Döring/Hoffmann 2012).

- Empirisch fundierte Aussagen über den Nutzen und die Effekte von Qualifizierungsberatung fehlen bislang. Damit sind eine Marktentwicklung bzw. die Identifikation eines "marktgängigen Produktes“ erschwert (Döring et al. 2008). - Es fehlt eine fundierte Aufarbeitung des Zusammenhanges zwischen Qualifizierungsberatung und Intensivierung der Weiterbildung; die unterstellte positive Korrelation ist bislang empirisch nicht belastbar (Döring/Rätzel 2007a).

- Ungeklärt ist bisher, ob und wie Unternehmensverantwortliche den Zusammenhang zwischen betrieblicher Modernisierung, Personalentwicklung und Beschäftigten einerseits und externer Beratung andererseits einschätzen und ob sie die Entwicklung einer betrieblichen Lernkultur als subjektive, organisationsbezogene und von Beratung abhängige Prozesskette zugleich sehen (Döring/Rätzel 2007a).

- In der Forschung ist bislang nur marginal geklärt, welche strategischen und qualitativen Anforderungen an eine inte- grierte Beratung zu stellen sind, die sowohl die Organisation (KMU als Betrieb) als auch die Beschäftigten (Individuum) zugleich in den Blick nimmt (Düsseldorff/Wohlfart 2012; Fischell 2012).

Angesichts der Forschungsdesiderate und der damit in $\mathrm{Zu}$ sammenhang stehenden Handlungsherausforderungen (Diekmann 2008; 2012) steht nach wie vor die Einschätzung im Raum, dass in der Regel Unternehmensverantwortliche den Zusammenhang zwischen betrieblicher Modernisierung, Qualifizierungsaktivitäten und vorgelagerten oder begleitenden Beratungsdienstleistungen nicht als kritischen Erfolgsfaktor für die Aktualisierung der betrieblichen Personalressourcen identifizieren. Insofern wird eine entsprechende externe Infrastrukturbildung von Qualifizierungsberatungsdienstleistungen durch die Marktakteure zwar nicht offensiv eingefordert, sie wird aber auf der politischprogrammatischen Ebene ohne nähere Konkretisierungsperspektiven thematisiert, wie beispielhaft am Empfehlungspapier des vom BMBF 2006 (BMBF 2008) eingerichteten Innovationskreises Weiterbildung zu belegen ist, der in seinem Abschlusspapier die Bildungs- und Qualifizierungsberatung für die Realisierung des Lernens im Lebensverlauf zwar als individuelle und betriebliche Schlüsselstrategie sieht, dafür aber keine konkreten strukturbildenden Maßnahmen bzw. Qualitätsstrategien und schon gar nicht unter öffentlicher Verantwortung benennt.

\section{Handlungsfelder einer Qualifizierungsberatung}

Im Kern der KMU-Qualifizierungsberatung geht es um die Organisation „KMU“, deren Schnittstellen zwischen personenbezogener und organisationsbezogener Beratung und zwischen betriebswirtschaftlicher und pädagogischer Ausrichtung nicht klar zu markieren sind (Düsseldorff 2009 et al., S. 38). Denn Qualifizierungsberatung unterstützt die Betriebe u. a.:

- bei der systematischen Personal- und Organisationsentwicklung,

- bei der Identifikation der Schnittstellen zwischen betrieblichen Veränderungsprozessen, wertschöpfungsbezogenen Wettbewerbsstrategien und Qualifizierung,

- speziell bei der Bildungs- und Qualifizierungsbedarfsanalyse angesichts veränderter interner und externer Rahmenbedingungen,

- bei der Planung, Durchführung und Evaluation betrieblicher Weiterbildung,

- bei der kontinuierlichen Entwicklung einer betrieblichen Lernkultur,

- bei der Integration bislang von Weiterbildung ausgeschlossener Beschäftigungsgruppen, 
- bei der Gestaltung geeigneter Lernwege, -methoden und -verfahren, eingeschlossen der Gestaltung lernförderlicher Arbeitsbedingungen

- und nicht zuletzt bei der Etablierung der betrieblichen Weiterbildung zu einem betrieblichen Funktionsfeld (Dobischat et al. 2008a).

Aus dieser Auflistung wird erkennbar, dass die Beratungsleistungen sowohl betriebswirtschaftlich als auch erziehungswissenschaftlich zu fundieren sind. Ziel einer umfassenden, integrierten KMU Qualifizierungsberatung ist letztlich eine Hilfestellung dafür, was unter der Begrifflichkeit „Betriebliches Weiterbildungsmanagement oder Bildungsmanagement" firmiert (Stender 2009, S. 42ff.; Diesner 2008).

Wie bereits ausgeführt, ist das Forschungsfeld bislang nur wenig konturiert, wobei nur wenige bzw. verstreute empirisch fundierte Studien vorliegen, die zudem auf kleinen Samples und nicht vergleichbaren Daten basieren. Illustrierend und exemplarisch ist daher auf einige neuere Ergebnisse aus Studien hinzuweisen, die den Kontext von Beratung aufgreifen und thematisieren. So geht aus einer Befragung hervor, dass „die Mobilisierung von Qualifikationsreserven von z. B. Älteren, Frauen, Migranten, Berufsrückkehrerinnen und An- und Ungelernten“ (Baderschneider et al. 2012, S. 51), Hilfen für den „Wissenstransfer zwischen alten und jungen Arbeitnehmern" und die Organisation der Weiterbildung als explizite Beratungsthemen „einen hohen Stellenwert bei den Unternehmen“ einnehmen. Insbesondere der Einsatz neuer Lernmedien, die Gestaltung variabler Lernwege und die Nutzung arbeitsplatznahen Lernens für Betriebe stellen einen hohen Beratungs- und Unterstützungsbedarf dar, der von Betrieben reklamiert wird (ebenda, S. 52, Tabelle 8).

Unabhängig von artikulierten Bedarfsthemen wird für das Thema „Beratung“" mehrheitlich von den Betrieben ein zukünftig wachsendes Bedarfsvolumen prognostiziert. Der Beratungsbedarf, so geht aus verschiedenen Studien hervor, erwartet unterschiedliche Beratungsformen, wobei die unmittelbare Beratung im Betrieb als zentrale Anforderung dominiert (ebenda, S. 53, Tabelle 9). Für die Bedarfsabgleichung und -artikulation, die mittels einer institutionalisierten „Beratungsstelle“ erfolgt, votieren nur wenige Betriebe.

Fasst man die spärlichen empirischen Befunde vor dem Hintergrund der derzeitigen Angebotssituation zusammen, so lässt sich einerseits ein hohes und anspruchsvolles Anforderungsprofil erkennen, andererseits gemessen daran aber nur eine unzureichende Praxis diagnostizieren: „So wie sich Qualifizierungsberatung derzeit darstellt, kann sie die Anforderungen nicht erfüllen, die an sie gestellt werden: eine dauerhafte Beratungsleistung für Betriebe zur Unterstützung einer systematischen Personalentwicklung" (Baderschneider et al. 2012, S. 60).

\section{Qualifizierungsberatung in öffentlicher Verantwortung?}

Die defizitäre Ausprägung einer auf Bildung, Beruf und Beschäftigung angelegten, professionalisierten und qualitativ und quantitativ in finanzieller und institutionell-organisatorisch gesicherten Beratung in Deutschland, die flächendeckende und anforderungsgerechte Angebote bereithält, existiert (noch) nicht, was die KMU-Modernisierung und Wettbewerbssicherung durch Weiterbildung einschränkt. „Bildungsberatung ist ein Strukturelement des Bildungswesen" - so hatte es bereits der Deutsche Bildungsrat in seinem Strukturplan (1970, S. 91) gefordert. Trotz des Anerkennens von Bildungsberatung als ein wichtiges Strukturelement im Bildungssystem wurde die Bildungsberatung bildungspolitisch in die Peripherie gedrängt und folglich nicht verbindlich öffentlich strukturiert und verantwortet.

Beratung spielt heute in unterschiedlichen Segmenten des Bildungssystems, wie die allgemeine Bildungsberatung als Schullaufbahnberatung, die Lernberatung oder schulpsychologische Beratung sowie als Beratung an der Schnittstelle zwischen Sekundarstufe I und II und Berufs- bzw. Studieneinmündung eine Rolle, in der Weiterbildung allgemein wie auch in der beruflichen Weiterbildung aber nicht (Schiersmann 2011; Wittwer 2006). Das betrifft auch die KMU-Qualifizierungs- und Bildungsberatung (Düsseldorff/ Wohlfart 2012), die seit der Veröffentlichung des Strukturplanes nicht ihren Platz in der Architektur des Bildungssystems gefunden hat. Ein im Auftrag des BMBF (Bundesministerium für Bildung und Forschung) veröffentlichtes Gutachten attestiert vielmehr dem deutschen Bildungssystem insgesamt eine Beratungslandschaft, die u. a. durch nicht systematisierte, marktförmig und öffentlich nur unzureichend verantwortete Strukturen eine entsprechend kontingente Versorgung mit zusätzlichen regionalen Disparitäten, eine Intransparenz der Angebote, Finanzierungs- und Qualitätsdefizite sowie durch überwiegend ungeregelte Professionalisierung auffällig wird (Rambóll Management 2008). Die daraus resultierenden umfänglichen Zugangs- und Versorgungsprobleme, so die Studie, sind weitreichend. Zwar hat - wiederum programmatisch - der vom BMBF einberufene Innovationskreis Weiterbildung (IKWB) im Gefolge der Studie formuliert: „Für kleine und mittlere Unternehmen sind Bildungsberatungsangebote auszubauen und weiterzuentwickeln“ (BMBF 2008, S. 18), aber noch im Jahr 2011 musste die Bundesregierung im Rahmen einer Kleinen Anfrage im Deutschen Bundestag einräumen, über eine Erprobung durch die Bundesagentur für Arbeit (BA) in Sachen KMU-Bildungs- und Qualifizierungsberatung nicht hinausgekommen zu sein (Deutscher Bundestag 2011): „Zudem erprobt die BA derzeit die Qualifizierungsberatung von KMU. Intention ist es, einen Beitrag zur Erhöhung betrieblicher Weiterbildungsaktivitäten im Hinblick auf den sich abzeichnenden demografischen Wandel zu leisten und dem Fachkräftemangel nachhaltig zu begegnen“ (ebenda, S. 8). 
In einem so gefassten Verständnis, dass erneut nur punktuell erprobt und nicht verbindlich etabliert wird, dürfte KMU Qualifizierungsberatung allerdings kaum einen Königsweg darstellen, denn sie sollte weit mehr sein als eine unterstützende Begleitung der Personalentwicklung mittelständischer Unternehmen durch die BA. Nur: Die Antwort in der Kleinen Anfrage und der Verweis auf die BA sind konsequent, denn die politischen Entscheidungsträger setzen gar nicht auf eine breite öffentlich strukturiert und verantwortete Lösung. Darüber klärt die Intention des mit dreijähriger Laufzeit (2010 bis 2012) vom BMBF geförderten Forschungsprojekts „MarQua - Strukturen und Strategien für eine marktfähige Qualifizierungsberatung “ auf. Es stellt aus der Sicht des Ministeriums einen wichtigen „Schritt in Richtung einer verbesserten Marktfähigkeit von Qualifizierungsberatung [... “" dar und zielt auf die Identifikation von Handlungsstrategien für die KMU-Qualifizierungsberatung, um diese „auf dem Markt besser zu positionieren“" (Loebe/Severing 2012a, S. 9f.).

\section{Fazit und Ausblick}

Die dargestellten Befunde deuten darauf hin, dass die Option, KMU zu einem Treiber für die Intensivierung der betrieblichen Weiterbildung zu entwickeln, bislang nicht zu den erwünschten Ergebnissen geführt hat. Denn weder ist es KMU in einem erkennbaren Ausmaß gelungen, lernförderliche Arbeitsbedingungen oder gezielte Investitionszuwächse in formelle betriebliche Weiterbildung zu realisieren und damit das Weiterbildungsverhalten insgesamt und besonders mit Blick auf Risikogruppen zu intensivieren, noch deuten die bislang nur zögerlichen Versuche, Qualifizierungsberatung unter öffentlicher Verantwortung als Stimulanz im oben angegebenen Sinne zu nutzen, auf eine Erfolgsperspektive hin.

Auch andere, hier nur angedeutete womöglich Erfolg versprechende Interventionsstrategien, wie öffentliche Förderprogramme im Rahmen der aktiven Arbeitsmarktpolitik und tarifvertragliche Vereinbarungen zwischen den Sozialpartnern, scheinen perspektivisch nur eingeschränkt aussichtsreich zu sein, da auch die angewandte Praxis in beiden Handlungsfeldern nicht sicherstellen konnte, weitreichende Ausgrenzungsrisiken in der beruflich-betrieblichen Weiterbildung zu minimieren und das Weiterbildungsengagement der KMU ansteigen zu lassen. Die Forderung nach der Etablierung einer mehr oder minder institutionalisierten Qualifizierungsberatung speziell für KMU als originär öffentliche Aufgabe bzw. in öffentlicher Verantwortung wird umstandslos mit heftiger Kritik konfrontiert werden, da die zentrale Frage der Finanzierung und Verortung angesprochen ist. In staatlicher Verantwortung hieße, die Finanzierung primär über Steuermittel zu garantieren, was bei gegenwärtiger Haushaltslage illusorisch erscheint. Eine kontinuierliche Beitragsfinanzierung über die Bundesagentur wie z. B. bei der Programmlinie WeGebAU dürfte zu Konflikten zwischen den Akteuren im Verwaltungsrat der Bundesagentur führen, da es bei einer auf bestimmte Aktionsfelder konzentrierten Förderung zu Wettbewerbsverzerrungen zwischen Marktteilnehmern führen kann. Da Qualifizierungsberatung für KMU ein wichtiges Aktionsfeld darstellt, ist also nach anderen Organisations- und Finanzierungsformen zu fragen. Diese müssten in die Richtung pluraler Beteiligungsformen gehen, wobei die Kammern wie auch die Gewerkschaften (z. B. über Fondlösungen bei der Qualifizierung) neben anderen Akteuren eine wichtige Funktion übernehmen könnten.
Letztlich, versucht man aus den skizzierten Ergebnissen Forschungsbedarfe zu bilanzieren, die den Kontext KMU und betriebliche Weiterbildung tiefer gehend analysieren und gegen den Mainstream der großbetrieblichen Weiterbildungsforschung orientiert wären, so sind Studien angezeigt, die die Ursachen der Weiterbildungszurückhaltung in ihrer Verbindung zu betrieblichen Entscheidungsprozessen gegen Weiterbildung fundiert klären und im Gegenzug die positiven Effekte einer aktiven betrieblichen KMU Personalpolitik mit dem Kernelement „Qualifizierung“ unter Kosten- und Nutzenaspekten deutlicher dokumentieren. Nicht zuletzt wäre auch herauszuarbeiten, ob und wie ein verstärktes öffentliches Engagement die KMUSituation in diesem Zusammenhang wirksam unterstützen könnte.

\section{LITERATUR}

Arnold, R./Mai, J. (2009): Bildungsberatung - historische Entwicklung und aktuelle Begriffsbestimmung, in: Arnold, R./Giesecke, W./Zeuner, C. (Hrsg.): Bildungsberatung im Dialog, Bd. 1, Baltmansweiler, S. 213-226

Autorengruppe Bildungsberichterstattung (Hrsg.) (2012): Bildung in Deutschland 2012. Ein indikatorengestützter Bericht mit einer Analyse zur kulturellen Bildung im Lebenslauf, Bielefeld

Baderschneider, A./Diller, F./Döring, O. (2012b): Qualifizierungsberatung auf dem Prüfstand: Status und Handlungsempfehlungen, in: Loebe, H./Severing, E. (Hrsg.): Qualifizierungsberatung in KMU, Bielefeld, S. 35-72

Bahnmüller, R. (2012): Tarifvertragliche Regulierung von Weiterbildung: An satzpunkte und Erfahrungen, in: http://www.bibb.de/dokumente/pdf/stst foko_120309_arbeitskraeftebedarf_im_demografischen_wandel_bahnmueller. pdf (letzter Zugriff: 07.03.2013)

Bahnmüller, R./Fischbach, S./Jentgens, B. (2006): Was nützen und was bewirken Qualifizierungstarifverträge?, in: WSI-Mitteilungen 59 (2), S. 71-78, http:// www.boeckler.de/wsimit_2006_02_bahnmuller.pdf

Behringer, F./Käpplinger, B. (2011): Arbeitsplatznahe Lernformen und Lernortvielfalt in der betrieblichen Weiterbildung. Wachsende Bedeutung in ganz Europa oder deutsches Spezifikum, in: Berufsbildung in Wissenschaft und Praxis 40 (1) , S. $15-19$

Bundesinstitut für Berufsbildung (BIBB) (Hrsg.) (2012): Datenreport zum Berufsbildungsbericht 2012. Informationen und Analysen zur Entwicklung der beruflichen Bildung, Bonn

Bundesministerium für Bildung und Forschung (BMBF) (2008): Empfehlungen des Innovationskreises Weiterbildung für eine Strategie zur Gestaltung des Lernens im Lebensverlauf, Berlin/Bonn

Bundesministerium für Bildung und Forschung (BMBF) (2011): Bestandsaufnahme und Konsistenzprüfung beruflicher Weiterbildungsförderung auf Bundes- und Länderebene, Bonn

Bundesministerium für Bildung und Forschung (BMBF) (2013): Weiterbildungsverhalten in Deutschland, AES 2012 Trendbericht, Bielefeld

Deutscher Bildungsrat (1970): Empfehlungen der Bildungskommission: Struk turplan für das Bildungswesen, Stuttgart

Deutscher Bundestag (2011): Antwort der Bundesregierung auf die Kleine Anfrage der Abgeordneten Dr. Ernst Rossmann, Katja Mast, Willi Brase, weiterer Abgeordneter und der Fraktion der SPD "Zur Situation der Berufs- und Bildungsberatung in Deutschland“: Deutscher Bundestag, 17. Wahlperiode, Drucksache 17/5717 vom 02.05.

Diekmann, K. (2008): Herausforderungen von betriebsnahen Beratungsleistungen zur Qualifizierung, in: Loebe, H./Severing, E. (Hrsg.): Stand und Perspektiven der Qualifizierungsberatung, Bielefeld, S. 93-107

Diekmann, K. (2012b): Marktfähigkeit von Qualifizierungsberatung - Ist die Beratung ein Zukunftsmodell?, in: Loebe,H./Severing, E. (Hrsg.), a. a. O., S. $251-270$

Diesner, I. (2008): Bildungsmanagement in Unternehmen, Wiesbaden Dobischat, R./Düsseldorff, K./Fischell, M.(2008a): Leitfaden zur Qualifizierungsberatung von kleinen und mittleren Unternehmen (KMU), Essen Dobischat, R./Düsseldorff, K./Fischell, M.(2008b): Qualifizierungsberatung für KMU. Stand der Forschung und Entwicklungsperspektiven. Gutachten im Auftrag der Lernenden Region Trier, Duisburg/Essen (Universitätsdruck, hektographiertes MS) 
Dobischat, R./Düsseldorff, K./Schurgatz, R. (2011): Beruflich-betriebliche Weiterbildung, in: Zeuner, Ch. (Hrsg.): Enzyklopädie Erziehungswissenschaft Online (EEO): Erwachsenenbildung, Weinheim/Basel

Döring, O./Hoffmann, J. (2012): Forschungs- und Entwicklungsstand der Weiterbildungs- und Qualifizierungsberatung, in: Loebe, H./Severing, E. (Hrsg.)

(2012b): a.a.O., S. 271-292

Döring, 0./Gottwald, M./Hinz, A./Löffelmann, S. (2008): Organisations- und Qualifizierungsberatung unter besonderer Berücksichtigung von Klein- und Mittelbetrieben, in: Loebe, H./Severing, E. (Hrsg.): a. a. O., S. 157-199

Döring, O./Rätzel, D. (2007a): Verbesserung der betrieblichen Weiterbildung durch Qualifizierungsberatung?, in: Zeitschrift für Berufs- und Wirtschaftspädagogik 102 (2), S. 196-212

Döring, O./Rätzel, D. (2007b): Aktuelle Aspekte von Qualifizierungsberatung für Betriebe, in: report 30 (2), S. 51-61.

Düsseldorff, K. (2006): Betriebliche Weiterbildung in KMU. Zum Stand der Forschung, in: Gonon, P./Klauser, F./Nickolaus, R. (Hrsg.): Kompetenz, Qualifikation und Weiterbildung im Berufsleben, Opladen, S. 179-193

Düsseldorff, K./Fischell, M./Scholz, J. (2009): Qualifizierungsberatung in KMU Eine Fallstudie aus dem Förderprogramm „Lernende Regionen - Förderung von Netzwerken“, in: Berufsbildung in Wissenschaft und Praxis 38 (4),

S. $38-40$

Düsseldorff, K./Wohlfart, U. (2012): Qualifizierungsberatung durch Strukturbil dung und Qualitätsentwicklung stärken, in: Loebe, H./Severing, E. (Hrsg.)

(2012b), a.a.O., S. 127-142

Elsholz, U. (2010): Arbeitsbezogenes Lernen in der beruflich-betrieblichen Weiterbildung als Beitrag zu mehr Chancengleichheit, in: Berufs- und Wirtschaftspädagogik - online, www.bwpat.de/ausgabe19/elsholz_bwpat10.pdf, (letzter Zugriff 10.01.2013)

Fischell, M. (2012): Betriebliche Qualifizierungsberatung als Tätigkeitsfeld Welchen Beitrag leistet die Professionalisierungsdebatte?, in: Loebe, H./Severing (Hrsg.) (2012b), a.a.O., S. 145-162

Gonon, P./Hotz, H.-P./Schläfli, A. (2004): Berufsbildung, Weiterbildung und KMU in der Schweiz, in: Gonon, P. /Stolz, S. (Hrsg.): Betriebliche Weiterbildung, Bern, S. 35-50

Gonon, P./Stolz, S. (Hrsg.) (2004): Betriebliche Weiterbildung, Bern Haak, C (2003): Weiterbildung in kleinen und mittleren Betrieben, Berlin Institut für Mittelstandsforschung (IfM) (2011): Kennzahlen zum Mittelstand 2008/2010 in Deutschland, Bonn

Jablonka, P. (2009): Bildungsscheck NRW. Evaluation eines Förderinstruments, in: DIE-Magazin (3), S. 45- 48

Käpplinger, B. (2007): Welche Betriebe in Deutschland sind weiterbildungsaktiv? Nutzung des CVTS-Datensatzes zur Analyse der betrieblichen Weiterbildung, in: Zeitschrift für Berufs- und Wirtschaftspädagogik 103 (3), S. 382-396 Kay, R./Kranzusch, P./Supinovic, O. (2008): Absatz- und Personalpolitik mittelständischer Unternehmen im Zeichen des demographischen Wandels - Herausforderungen und Reaktionen: Institut für Mittelstandsforschung, IfM-Materialien (183), Bonn

Koscheck, S. (2010): Bildungsgutscheine: Auswirkungen auf Angebot und wirtschaftliche Situation von Weiterbildungsanbietern in Ost und West, in: Berufsbildung in Wissenschaft und Praxis 39 (4), S. 4-5

Leber, U. (2009): Betriebsgröße, Qualifikationsstruktur und Weiterbildungsbeteiligung - Ergebnisse aus dem IAB-Betriebspanel, in: Behringer, F./Käpplinger, B./Pätzold, G. (Hrsg.): Betriebliche Weiterbildung, Beiheft 22 der ZBW, Stuttgart, S. $149-168$

Loebe,H./Severing, E (2008) (Hrsg.): Stand und Perspektiven der Qualifizierungsberatung, Bielefeld

Loebe, H./Severing, E. (2011) (Hrsg.): Der Markt für Qualifizierungsberatung Fiktion, Realität, Vision?, Bielefeld
Loebe, H./Severing, E. (2012a): Einleitung - Förderung systematischer Personalentwicklung in KMU durch Qualifizierungsberatung, in: Dieselben (Hrsg.) (2012b), S. 7-14

Loebe, H./Severing, E. (2012b) (Hrsg.): Qualifizierungsberatung in KMU, Bielefeld

Lott, M./Spitznagel, E. (2007): Wenig Betrieb auf neuen Wegen der beruflichen Weiterbildung, IAB-Kurzbericht 23/2007, Nürnberg

Lott, M./Spitznagel, E. (2010): Präventive Arbeitsmarktpolitik: Impulse für die berufliche Weiterbildung im Betrieb, IAB-Kurzbericht 11/2007, Nürnberg Möller, J/Walwei, U. (Hrsg.) (2009): Handbuch Arbeitsmarkt, Nürnberg Moraal, D./Schönfeld, G. (2012): Berufliche Aus- und Weiterbildung in Unternehmen, in: WSI-Mitteilungen 65 (5), S. 329-337, http://www.boeckler.de/ wsimit_2012_05_Moraal.pdf

Munk, P. (2012): Vorwort, in: Loebe, H./Severing, E. (Hrsg.) (2012b), a. a. O., S. 5-6 Neubäumer, R./Kohaut, S./Seidenspinner, M. (2006): Determinanten der betrieblichen Weiterbildung. Ein ganzheitlicher Ansatz zur Erklärung des betrieblichen Weiterbildungsverhaltens und eine empirische Analyse für Westdeutschland, in: Schmollers Jahrbuch für Wirtschafts- und

Sozialwissenschaften 126 (3), S. 437-471

Rambóll Management (2007): Bestandsaufnahme in der Bildungs-, Berufsund Beschäftigungsberatung und Entwicklung grundlegender Qualitätsstandards. Abschlussbericht, hrsg. im Auftrag des BMBF, Bonn/Berlin

Richter, M. (2009): Mittelständische Personalpolitik: Charakteristika, Problemfelder und Handlungsempfehlungen. Gutachten im Auftrag des Arbeitskreis Mittelstand der Friedrich-Ebert-Stiftung, Bonn

Schiersmann, C. (2011): Beratung im Kontext lebenslangen Lernens, in:Tippelt, R./von Hippel, A. (Hrsg.): Handbuch Erwachsenenbildung/Weiterbildung, Wiesbaden, S. 747-767

Stegmeier, J. (2010): Betriebliche Aus und Weiterbildung in der Krise, in: Berufsbildung in Wissenschaft und Praxis 39 (6), S. 4-5

Staudt, E. (1993): Personalentwicklung für die neue Fabrik, Opladen

Stender, J. (2009): Betriebliches Weiterbildungsmanagement, Stuttgart

Vages, G. (2012): Kompetenzsicherung im Mittelstand, in: Berufsbildung 66 (138), S. 20-24

Wittwer, W. (2006): Berufliche Weiterbildungsberatung, in: Faulstich, P./Bayer, M. (Hrsg.): Lernwiderstände. Anlässe für Vermittlung und Beratung, Hamburg, S. $90-110$

\section{AUTOREN}

ROLF DOBISCHAT, Dr., Professor für Wirtschaftspädagogik am Institut für Berufs- und Weiterbildung der Universität Duisburg-Essen. Arbeitsschwerpunkt: Regionale Berufs- und Weiterbildungsforschung

rolf. dobischat@uni-due.de

KARL DÜSSELDORFF, Dr., apl-Professor am Institut für Berufs- und Weiterbildung der Universität Duisburg-Essen. Arbeitsschwerpunkt: Betriebliche Weiterbildungsforschung.

karl.duesseldorff@uni-due.de 